PROCEEDINGS OF THE

AMERICAN MATHEMATICAL SOCIETY

Volume 130, Number 7, Pages 1881-1886

S 0002-9939(02)06532-2

Article electronically published on February 27, 2002

\title{
CONVERGENCE OF ZETA FUNCTIONS OF GRAPHS
}

\author{
BRYAN CLAIR AND SHAHRIAR MOKHTARI-SHARGHI
}

(Communicated by Dennis A. Hejhal)

\begin{abstract}
The $L^{2}$-zeta function of an infinite graph $Y$ (defined previously in a ball around zero) has an analytic extension. For a tower of finite graphs covered by $Y$, the normalized zeta functions of the finite graphs converge to the $L^{2}$-zeta function of $Y$.
\end{abstract}

\section{INTRODUCTION}

Associated to any finite graph $X$ there is a zeta function $Z(X, u), u \in \mathbb{C}$. It is defined as an infinite product but shown (in various different cases) by Ihara, Hashimoto, and Bass [5, 4, 1. to be the reciprocal of a polynomial. Indeed the rationality formula for a $q+1$ regular $X$ states that:

$$
Z(X, u)^{-1}=\left(1-u^{2}\right)^{-\chi(X)} \operatorname{Det}\left(I-\delta u+q u^{2}\right) .
$$

Here $\delta$ is the adjacency operator of $X$.

In [2], an $L^{2}$-zeta function is defined for noncompact graphs with symmetries, using the machinery of von Neumann algebras. A rationality formula similar to (0.1) expresses the relationship between the zeta function and the von Neumann determinant of a Laplace operator. The results of this paper focus on a special case. Let $Y$ be an infinite graph which covers a finite graph $B=\pi \backslash Y$. The $L^{2}$-zeta function $Z_{\pi}(Y, u)$ is defined in [2] only in a small neighborhood of zero. The first result of this paper is to extend the $L^{2}$-zeta function to the interior of $C=\left\{u \in \mathbb{C}:|u|=q^{-1 / 2}\right\} \cup\left[-1,-\frac{1}{q}\right] \cup\left[\frac{1}{q}, 1\right]$.

In the second part of the paper, we consider a tower of finite graphs $B_{i}$ covered by $Y$. Put $N_{i}=\left|B_{i}\right| /|B|$. In Theorem 2.1] we show that the zeta functions for the $B_{i}$, renormalized by taking $N_{i}^{\text {th }}$ roots, converge to the $L^{2}$-zeta function for $Y$. The argument is inspired by, and uses, work of Lück [7].

In the first section we recall the definitions of the zeta functions of finite and infinite graphs. One of the main results of this paper is Theorem 1.5 in this section. In the second section of this paper we prove the convergence theorem and exhibit interesting examples. Theorem [2.5] generalizes work of Deitmar [3].

\section{ZETA FUNCTIONS}

In this section we recall the definition of the zeta function and related material. We first recall the definition of the zeta function for finite graphs.

Received by the editors August 4, 2000.

2000 Mathematics Subject Classification. Primary 11M41; Secondary 05C25.

(C)2002 American Mathematical Society 
1.1. Finite graphs. For a graph $X$, let $V X$ and $E X$ denote the sets of vertices and edges, respectively, of $X$. If each vertex has the same degree, then $X$ is regular.

Definition 1.1. Let $X$ be a finite graph. A closed path in $X$ is primitive if it is not a nontrivial power of another path inside the fundamental group of $X$. Let $P$ be the set of free homotopy classes of primitive closed paths of $X$. Then the zeta function of $X$ is

$$
Z(X, u)=\prod_{\gamma \in P}\left(1-u^{\ell(\gamma)}\right)^{-1},
$$

where $\ell(\gamma)$ is the minimum length of paths in the class of $\gamma$.

Let $\delta$ be the adjacency operator of the graph $X$ acting on $l^{2}(V X)$. For $f \in$ $l^{2}(V X)$ let $Q f(x)=q(x) f(x)$ where $q(x)+1$ is the degree of the vertex $x$. Put $\Delta(X, u)=I-\delta u+Q u^{2}$. The Ihara rationality formula says that $Z(X, u)^{-1}$ is a polynomial:

$$
Z(X, u)^{-1}=\left(1-u^{2}\right)^{-\chi(X)} \operatorname{Det}(\Delta(X, u))
$$

The zeta function satisfies the following functional equation [1, Corollary 3.10]:

$$
Z\left(X,(q u)^{-1}\right)=\left(\frac{1-u^{2}}{q^{2} u^{2}-1}\right)^{-\chi(X)} q^{2 e-v} u^{2 e} Z(X, u)
$$

where $e=|E X|, v=|V X|$ and $\chi(X)$ is the Euler characteristic of $X$.

For more details and examples see 9,10 .

The following proposition is well known. A proof can be found in [6, page 59].

Proposition 1.2. The poles of the zeta function for any finite $q+1$ regular graph lie on the set $C$ (Figure 1) where

$$
C=\left\{u \in \mathbb{C}:|u|=q^{-1 / 2}\right\} \cup\left[-1,-\frac{1}{q}\right] \cup\left[\frac{1}{q}, 1\right] .
$$

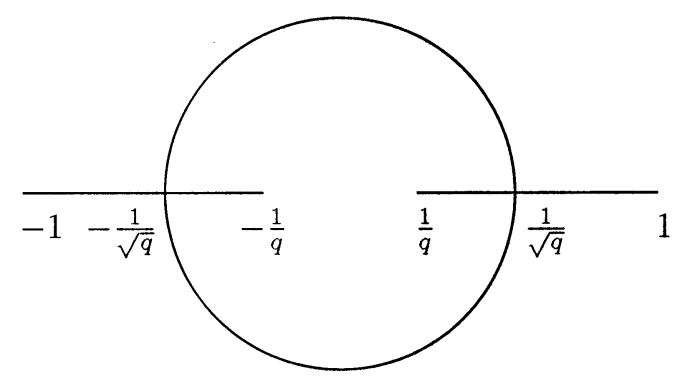

Figure 1. The set $C$

Let $\Omega$ be the interior of $C$. Then:

Proposition 1.3. If $u \in \Omega$ and $\lambda \in[-(q+1), q+1]$, then $1-\lambda u+q u^{2} \in \mathbb{C}-(-\infty, 0]$.

Proof. Let $u=a+b i$. For $1-\lambda u+q u^{2}$ to be real we must have $1-\lambda u+$ $q u^{2}=1-\lambda \bar{u}+q \bar{u}^{2}$. This implies that either $b=0$ or $a=\frac{\lambda}{2 q}$. If $a=\frac{\lambda}{2 q}$, then $1-\lambda u+q u^{2}=1-q\left(a^{2}+b^{2}\right)$ which is negative or zero if $u$ is on or outside of the circle $|u|=q^{-1 / 2}$. In the case that $b=0$ put $f(a):=1-\lambda a+q a^{2}=1-\lambda u+q u^{2}$. If $f$ has no real root, then $f$ is always positive. Otherwise $|\lambda| \geq 2 \sqrt{q}$. Then $f\left(-\frac{1}{q}\right)$ and 
$f\left(\frac{1}{q}\right)$ are nonnegative, and $f^{\prime}$ is not zero in $\left(-\frac{1}{q}, \frac{1}{q}\right)$. Therefore $f$ will be positive on $\left(-\frac{1}{q}, \frac{1}{q}\right)$.

Corollary 1.4. For a $q+1$ regular finite graph $X$, the polynomial Det $\Delta(X, u)$ has an analytic $N^{\text {th }}$ root on $\Omega$ for all $N \in \mathbb{N}$.

Proof. We know Det $\Delta(X, u)=\Pi_{\lambda}\left(1-\lambda u+q u^{2}\right)$ where $\lambda$ varies over eigenvalues of the adjacency operator of the graph. Then $\Pi_{\lambda}\left(1-\lambda u+q u^{2}\right)^{\frac{1}{N}}$ is an analytic $N^{\text {th }}$ root for Det $\Delta(X, u)$.

1.2. Infinite graphs. For $\pi$ a countable discrete group, the von Neumann algebra of $\pi$ is the algebra $\mathcal{N}(\pi)$ of bounded $\pi$-equivariant operators from $l^{2}(\pi)$ to $l^{2}(\pi)$. The von Neumann trace of an element $f \in \mathcal{N}(\pi)$ is defined by

$$
\operatorname{Tr}_{\pi} f=\langle f(e), e\rangle
$$

for $e \in \pi$ the unit element. For $H=\bigoplus_{i=1}^{n} l^{2}(\pi)$ and a bounded $\pi$-equivariant operator $f: H \rightarrow H$, define

$$
\operatorname{Tr}_{\pi} f=\sum_{i=1}^{n} \operatorname{Tr}_{\pi} f_{i i}
$$

The trace as defined is independent of the decomposition of $H$. The von Neumann trace extends to bounded $\pi$-equivariant operators on Hilbert $\mathcal{N}(\pi)$-modules, but they will not be needed.

Now let $Y=(V Y, E Y)$ be an infinite graph. Suppose the group $\pi$ acts freely on $Y$ with finite quotient $B$.

Let $P$ denote the set of free homotopy classes of primitive closed paths in $Y$. For $\gamma \in P, \ell(\gamma)$ is the length of the shortest representative of $\gamma$. The group $\pi_{\gamma}$ is the stabilizer of $\gamma$ under the action of $\pi$. The $L^{2}$-zeta function of $Y$ is the infinite product

$$
Z_{\pi}(Y, u)=\prod_{\gamma \in \pi \backslash P}\left(1-u^{\ell(\gamma)}\right)^{-\frac{1}{\mid \pi \gamma}} .
$$

This definition comes from [2], but beware that in [2] the notation $Z_{\pi}(Y, u)$ refers to the reciprocal of the zeta function as defined here.

The adjacency operator $\delta$ and Laplace operator $\Delta(Y, u)=I-\delta u+Q u^{2}$ are $\pi$-equivariant operators on $l^{2}(V Y)$. Choosing lifts of vertices of $B$ yields a decomposition $l^{2}(V Y)=\bigoplus l^{2}(\pi)$. Then from [2, Theorem 0.3],

$$
Z_{\pi}(Y, u)^{-1}=\left(1-u^{2}\right)^{-\chi^{(2)}(Y)} \operatorname{Det}_{\pi} \Delta(Y, u) .
$$

In this formula, $\chi^{(2)}(Y)$ is the $L^{2}$-Euler characteristic of $Y$, which in our setting is simply equal to $\chi(B)$. The determinant $\operatorname{Det}_{\pi} \Delta(Y, u)$ is defined via formal power series as $\left(\operatorname{Exp} \circ \operatorname{Tr}_{\pi} \circ \log \right) \Delta(Y, u)$ and converges for small $u \in \mathbb{C}$. More precisely, if $Y$ is $q+1$ regular, then the radius of convergence of $Z_{\pi}(Y, u)$ is greater than or equal to $\frac{1}{q}$.

Theorem 1.5. Let $Y$ be a $q+1$ regular graph. Then $Z_{\pi}(Y, u)$ has a holomorphic extension to $\Omega$.

Proof. By (1.3) it is enough to show that $\operatorname{Det}_{\pi} \Delta(Y, u)$ has a nonzero holomorphic extension on $\Omega$. Let $g_{u}(\lambda)=\log \left(1-\lambda u+q u^{2}\right)$. Here and in the rest of the paper $\log$ is the principal branch of the logarithm, defined and analytic on $\mathbb{C}-(-\infty, 0]$. Fix 
$u \in \Omega$. Then using Proposition 1.3, there exists an open set $V_{u} \supset[-(q+1), q+1]$ on which $g_{u}$ is analytic. Since $\delta$ is self-adjoint and $\|\delta\| \leq q+1$, the spectrum $\sigma(\delta) \subset[-(q+1), q+1]$. By the spectral theorem for self-adjoint operators we can write:

$$
\delta=\int_{-k^{-}}^{k} \lambda d E(\lambda)
$$

Now

$$
g_{u}(\delta)=\int_{-k^{-}}^{k} \log \left(1-\lambda u+q u^{2}\right) d E(\lambda)
$$

is well defined, and

$$
\operatorname{Tr}_{\pi} g_{u}(\delta)=\int_{-k^{-}}^{k} \log \left(1-\lambda u+q u^{2}\right) d\left(\operatorname{Tr}_{\pi} E(\lambda)\right)
$$

is a holomorphic function of $u$ on $\Omega$. Now for small $u$,

$$
\begin{aligned}
\operatorname{Det}_{\pi}(\Delta(Y, u)) & =\operatorname{Exp} \operatorname{Tr}_{\pi} \log (\Delta(Y, u)) \\
& =\operatorname{Exp} \operatorname{Tr}_{\pi} \log \left(I-\delta u+q u^{2}\right) \\
& =\operatorname{Exp} \operatorname{Tr}_{\pi} g_{u}(\delta) .
\end{aligned}
$$

Remark. Using the functional equation (1.2) it is possible to extend the $L^{2}$-zeta function to the exterior of $C$. Defining the $L^{2}$-zeta function on $C$ itself presents some problems. In examples, the function on the interior of $C$ and the exterior of $C$ do not match continuously on $C$. However the absolute value of the resulting function is the Fuglede-Kadison determinant, and may extend continuously to $C$.

\section{Convergence of zeta FunCtions for towers of GRAPHS}

In this section we prove that the zeta functions for a tower of finite graphs, suitably renormalized, converge to the $L^{2}$-zeta function for an infinite covering graph. The argument uses an idea from [7].

\subsection{The convergence theorem.}

Theorem 2.1. Let $Y$ be a $q+1$ regular graph. Suppose the group $\pi$ acts freely on $Y$, and that $B=\pi \backslash Y$ is a finite graph. Suppose $\pi=\pi_{1} \supset \pi_{2} \supset \cdots$ is a tower of finite index normal subgroups and $\bigcap \pi_{i}=\{e\}$. Let $\left[\pi: \pi_{i}\right]=N_{i}$ and $B_{i}=\pi_{i} \backslash Y$. Then for $u \in \Omega$, we have

$$
\lim _{i \rightarrow \infty} Z\left(B_{i}, u\right)^{\frac{1}{N_{i}}}=Z_{\pi}(Y, u)
$$

The convergence is uniform on compact subsets of $\Omega$.

Remark. Notice that

$$
\begin{aligned}
Z\left(B_{i}, u\right)^{-1} & =\left(1-u^{2}\right)^{-\chi\left(B_{i}\right)} \operatorname{Det} \Delta\left(B_{i}, u\right) \\
& =\left(1-u^{2}\right)^{-N_{i} \chi(B)} \operatorname{Det} \Delta\left(B_{i}, u\right) .
\end{aligned}
$$

So we let

$$
Z\left(B_{i}, u\right)^{-1 / N_{i}}=\left(1-u^{2}\right)^{-\chi(B)}\left(\operatorname{Det} \Delta\left(B_{i}, u\right)\right)^{1 / N_{i}} .
$$

The $N^{\text {th }}$ root in (2.4) is taken in the sense of Corollary 1.4 . 
Proof of Theorem [2.1. From the remark we need to show that

$$
\lim _{i \rightarrow \infty}\left(\operatorname{Det} \Delta\left(B_{i}, u\right)\right)^{1 / N_{i}}=\operatorname{Det} \Delta(B, u) .
$$

Let $F_{i}(\lambda)=\frac{1}{N_{i}} \mid\left\{\mu\right.$ eigenvalue of $\left.\delta_{i}, \mu \leq \lambda\right\} \mid$. Let $F(\lambda)=\operatorname{Tr}_{\pi} E(\lambda)$, where $\{E(\lambda)\}_{\lambda}$ is the spectral decomposition of $\delta$ acting on $l^{2}(Y)$. We now set

$$
\begin{aligned}
\bar{F}(\lambda) & =\limsup _{i \rightarrow \infty} F_{i}(\lambda) ; \quad \underline{F}(\lambda)=\liminf _{i \rightarrow \infty} F_{i}(\lambda) ; \\
\bar{F}^{+}(\lambda) & =\lim _{\varepsilon \rightarrow 0^{+}} \bar{F}(\lambda+\varepsilon) ; \quad \underline{F}^{+}(\lambda)=\lim _{\varepsilon \rightarrow 0^{+}} \underline{F}(\lambda+\varepsilon) .
\end{aligned}
$$

Then from [7, Theorem 2.3.1], for all $\lambda \in[-k, k]$,

$$
F(\lambda)=\bar{F}^{+}(\lambda)=\underline{F}^{+}(\lambda) \text {. }
$$

We know

$$
\log \operatorname{Det} \Delta\left(Y_{i}, u\right)=\int_{-k^{-}}^{k} \log \left(1-u \lambda+q u^{2}\right) d F_{i}(\lambda) .
$$

By (1.6)

$$
\log \operatorname{Det}_{\pi} \Delta(Y, u)=\int_{-k^{-}}^{k} \log \left(1-u \lambda+q u^{2}\right) d F(\lambda) .
$$

If $K \subset \Omega$ is compact, then $\log \left(1-u \lambda+q u^{2}\right)$ is bounded uniformly for $u \in K$ and $\lambda$ in an open interval containing $[-k, k]$. Now integration by parts shows that indeed

$$
\int_{-k^{-}}^{k} \log \left(1-u \lambda+q u^{2}\right) d F_{i}(\lambda) \rightarrow \int_{-k^{-}}^{k} \log \left(1-u \lambda+q u^{2}\right) d F(\lambda)
$$

as $i \rightarrow \infty$.

\subsection{Examples.}

Example 2.2. Let $Y$ be the graph with vertices $\mathbb{Z}$ and edges connecting $n$ to $n+1$. The group $\pi=\mathbb{Z}$ acts on $Y$ with quotient having one vertex and one edge. Let $\pi_{n}=n \mathbb{Z} \subset \mathbb{Z}$, so $B_{n}=\pi_{n} \backslash Y$ is an $n$-cycle. As $B_{n}$ has only two primitive loops and $Y$ has none, we have $Z\left(B_{n}, u\right)=\left(1-u^{n}\right)^{-2}$ and $Z_{\pi}(Y, u)=1$. The graphs are 2-regular, so $\Omega$ is the (open) unit disk. For $u \in \Omega$,

$$
\lim _{n \rightarrow \infty}\left(1-u^{n}\right)^{2 / n}=1 .
$$

Notice that the functional equation (1.2) gives $Z_{\pi}(Y, u)=u^{-2}$ outside of the unit disk.

Example 2.3. Let $\pi=\mathbb{Z} \times \mathbb{Z}$, and let $Y$ be the Cayley graph of $\pi$ with generators $(1,0)$ and $(0,1) . Y$ is an infinite grid. We take for $\left\{\pi_{i}\right\}$ any nested sequence of $\mathbb{Z} \times \mathbb{Z}$ 's with $\bigcap \pi_{i}=\{e\}$. You can think of the quotient graphs $B_{i}$ as finite grids wrapped around tori. Then Theorem 2.1 gives that

$$
\begin{aligned}
\lim _{i \rightarrow \infty} \frac{1}{i} \log Z\left(B_{i}, u\right) & =\log Z_{\pi}(Y, u) \\
& =\sum_{M=1}^{\infty}\left[\frac{1}{M}+\sum_{d=0}^{M} \frac{(-3)^{M-d}}{M+d}\left(\begin{array}{c}
M+d \\
M-d
\end{array}\right)\left(\begin{array}{c}
2 d \\
d
\end{array}\right)^{2}\right] u^{2 M}
\end{aligned}
$$

as computed in [2]. 
Example 2.4. Let $Y$ be the $q+1$ regular tree and $B=\pi \backslash Y$ finite. Since $Y$ has no closed loops, the $L^{2}$-zeta function of $Y$ is the constant function 1. So if $B_{n}=\pi_{n} \backslash Y$ is a tower of finite graphs covering $B$,

$$
\lim _{n \rightarrow \infty} Z\left(B_{n}, u\right)^{1 /\left[\pi: \pi_{n}\right]}=1
$$

for $u \in \Omega$. This result is contained in [3] for $|u|$ small.

Theorem 2.5. Let $Y$ and $B$ be as in the previous example. We have:

$$
Z(B, u)=\frac{\operatorname{Det}_{\pi} \Delta(Y, u)}{\operatorname{Det} \Delta(B, u)}
$$

for $u \in \Omega$.

For small $u$, the above theorem is the main result of [3].

Proof. From [2, Theorem 0.3] we know that

$$
Z_{\pi}(Y, u)^{-1}=\left(1-u^{2}\right)^{-\chi^{(2)}(Y)} \operatorname{Det}_{\pi} \Delta(Y, u) .
$$

As in the previous example, $Z_{\pi}(Y, u)=1$. Now we have

$$
Z(B, u)=\frac{Z(B, u)}{Z_{\pi}(Y, u)}=\frac{\left(1-u^{2}\right)^{-\chi^{(2)}(Y)} \operatorname{Det}_{\pi} \Delta(Y, u)}{\left(1-u^{2}\right)^{-\chi(B)} \operatorname{Det} \Delta(B, u)} .
$$

As $\chi(B)=\chi^{(2)}(Y)$, the theorem follows.

\section{REFERENCES}

[1] H. Bass. Ihara-Selberg zeta function of a tree lattice. Int. J. of Mathematics, 3(6):717-797, 1992. MR 94a:11072

[2] Bryan Clair and Shahriar Mokhtari-Sharghi. Zeta functions of discrete groups acting on trees. J. Algebra, 237(2):591-620, 2001. CMP 2001:09

[3] Anton Deitmar. Combinatorial $L^{2}$-determinants. Proc. Edinburgh Math. Soc., 43(1):185-194, 2000. MR 2001f:11148

[4] K. Hashimoto. Zeta functions of finite graphs and representations of $p$-adic groups. In $A u$ tomorphic Forms and Geometry of Arithmetic Varieties, volume 15 of Adv. Studies in Pure Mathematics. Academic Press, 1989. MR 91i:11057

[5] T. Ihara. Discrete subgroups of $P L\left(2, k_{p}\right)$. In Proc. Symp. Pure Math. IX, pages 272-278. AMS, 1968. MR 34:5777

[6] Alexander Lubotzky. Discrete Groups, Expanding Graphs and Invariant Measures. Birkhäuser, 1994. MR 96g:22018

[7] Wolfgang Lück. Approximating $L^{2}$-invariants by their finite dimensional analogues. Geom. and Func. Anal., 4(4):455-481, 1994. MR 95g:58234

[8] Hirofumi Nagoshi. On arithmetic infinite graphs. Proc. Japan Acad., 76:22-25, 2000. MR 2001f:11150

[9] H.M. Stark and A.A. Terras. Zeta functions of finite graphs and coverings. Advances in Math., 121:124-165, 1996. MR 98b:11094

[10] H.M. Stark and A.A. Terras. Zeta functions of finite graphs and coverings, Part II. Advances in Math., 154:132-195, 2000. CMP 2000:17

Department of Mathematics, Saint Louis University, 220 N. Grand Ave., St. Louis, Missouri 63103

E-mail address: bryan@slu.edu

Department of Mathematics, Long Island University, Brooklyn Campus, 1 University Plaza, Brooklyn, New York 11201

E-mail address: mokhtari@liu.edu 